EESTI NSV TEADUSTE AKADEEMIA TOIMETISED. 28. KOIDE FOUSIKA * MATEMAATIKA. 1979, NR. 1

ИЗВЕСТИЯ АКАДЕМИИ НАУК ЭСТОНСКОИ ССР. ТОМ 28 ФИЗИКА * МАТЕМАТИКА. 1979, № 1

\title{
ТЕОРЕТИЧЕСКИЙ СИНТЕЗ ИНТЕРФЕРЕНЦИОННОГО ПОКРЫТИЯ С ДВУМЯ УЗКИМИ ПОЛОСАМИ ПРОПУСКАНИЯ
}

\author{
(Представлена П. Кардом)
}

Синтез светофильтров с одной узкой полосой пропускания является уже решенной задачей. В этом плане речь может идти только о дальнейшем усовершенствовании метода. Напротив, синтез светофильтров с несколькими узкими полосами пропускания при заданных длинах волн до сих пор исчерпывающим образом не осуществлен. В данной работе и предлагается эффективный метод синтеза таких фильтров. Этот метод настолько общ, что его можно распространить на синтез фильтров с любым числом узких полос пропускания. Здесь мы покажем его применение к синтезу фильтра с двумя полосами пропускания.

Будем исходить из общего метода синтеза интерференционных покрытий, изложенного в $\left[{ }^{1,2}\right]$. В этих работах показано, что заданная зависимость величины $A(k)=R(k) / T(k) \quad(R(k)$ и $T(k)$ - коэффициенты отражения и пропускания соответственно, $k$ - волновое число) может быть реализована неоднородным слоем с показателем преломления $n(x)$, зависящим от удвоенного оптического пути света $x$, согласно формуле

$$
n(x)=n(0) \exp \left\{\frac{2}{\pi}\left[\int_{0}^{\infty} \frac{P(k)}{k} \sin k x d k+\int_{0}^{\infty} \frac{Q(k)}{k}(1-\cos k x) d k\right]\right\} .
$$

Здесь $P(k)$ и $Q(k)$ - любые четная и нечетная функции, удовлетворяющие соотношению

$$
P^{2}(k)+Q^{2}(k)=A(k) .
$$

Коэффициент пропускания $T(k)$ выражается через характеристику $A(k)$, в силу равенства $R+T=1$, формулой

$$
T=1 /(A+1) \text {. }
$$

Отсюда видно, что для получения фильтра с несколькими полосами пропускания в заданных точках спектра нужно, чтобы $A$ было возможно большим везде, кроме точек, где находятся полосы пропускания. В этих точках должно быть $A=0$.

Учитывая формулу (2), видим, что этим требованиям наилучшим образом удовлетворяет выбор $P(k)$ и $Q(k)$ в внде скачкообразно изменяющихся ступенчатых функций, графики которых пересекают ось $k$ в точках пропускания. 


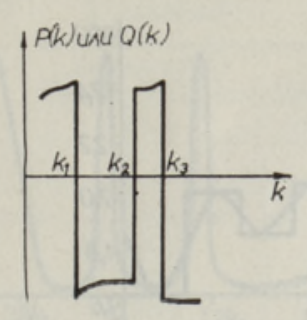

Рис. 1.

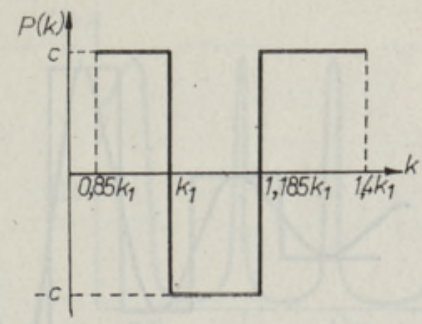

Рис. 2.

Между этими точками $P(k)$ и $Q(k)$ должны иметь большие значения, в остальном же их поведение несущественно; в частности, они могут быть постоянными, хотя это не обязательно. В любом случае большие значения $P(k)$ и $Q(k)$ гарантируют близость $T(k)$ к нулю (см. рис. 1).

После того как $P(k)$ и $Q(k)$ тем или иным образом выбраны, можем по формуле (1) найти $n(x)$, т. е. показатель преломления неоднородного слоя, реализующего требуемую спектральную характеристику.

\section{Синтез}

Применим теперь изложенный метод к синтезу светофильтра с двумя узкими полосами пропускания. Примем отношение пропускаемых частот равным 1,185 и обозначим волновые числа, при которых должны находиться полосы пропускания, через $k_{1}$ и $1,185 k_{1}$.

Выберем

$$
Q(k)=0
$$

и положим (см. рис. 2)

$$
P(k)=\left\{\begin{aligned}
C, \text { если } 0,85 k_{1} \leqslant k<k_{1}, 1,185 k_{1}<k<1,4 k_{1} ; \\
0, \text { если } k=k_{1}, k=1,185 k_{1} ; \\
-C, \text { если } k_{1}<k<1,185 k_{1} .
\end{aligned}\right.
$$

Этот выбор означает, что областью высокого отражения должен стать интервал [0,85 $\left.k_{1} ; 1,4 k_{1}\right]$.

Вычисляя по формуле (1) $n(x)$, находим

$$
\begin{aligned}
n(x)= & n(0) \exp \left\{\frac { 2 C } { \pi } \left[-\operatorname{Si}\left(0,85 k_{1} x\right)+2 \operatorname{Si}\left(k_{1} x\right)-\right.\right. \\
& \left.\left.-2 \operatorname{Si}\left(1,185 k_{1} x\right)+\operatorname{Si}\left(1,4 k_{1} x\right)\right]\right\} .
\end{aligned}
$$

Перейдем ко второму этапу синтеза, т. е., согласно $\left[{ }^{1,2}\right]$, к аппроксимации функции $n(x)$ ступенчатой кривой, наиболее точно имитирующей ход $n(x)$. Полученная в результате аппроксимации дискретно-слоистая последовательность должна приближенно реализовать заданную характеристику.

Если в формуле (6) примем $n(0)=1,86$ и $2 C / \pi=2$, то $n(x)$ получит вид, изображенный на рис. $3(x<0)$ и на рис. $4(x>0)$, причем $n(x)=n(0) / n(-x)$. На этих же рисунках показана и аппроксимация $n(x)$ ступенчатой кривой. До $\left|k_{1} x\right|<30$ аппроксимация проводится ступенчатой кривой, а после $\left|k_{1} x\right|>30$ показатель преломления изменяется мало и поэтому принимается постоянным. Получается 20-слой- 
Рис. 3.

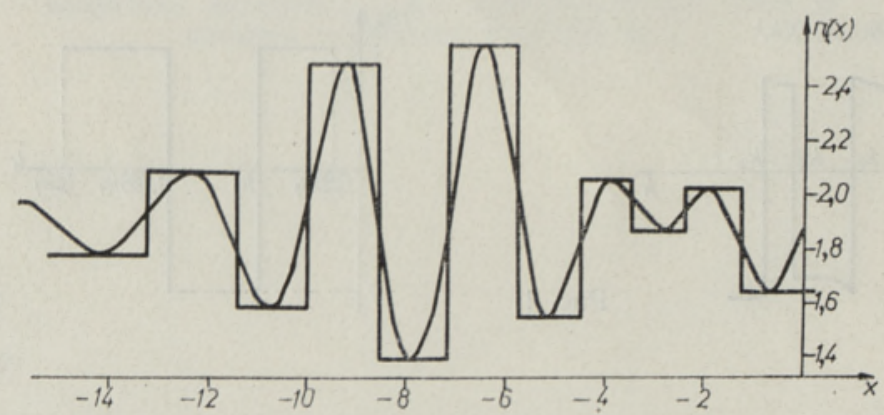

Рис. 4.

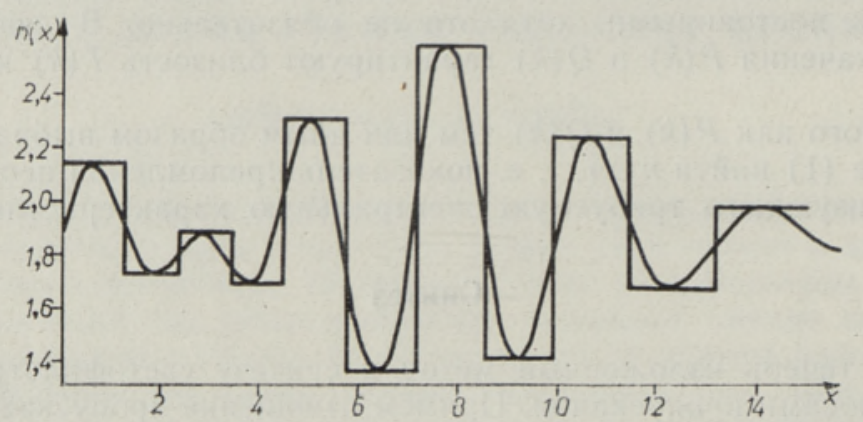

Таблица 1

\begin{tabular}{r|c|c|c}
\hline $\begin{array}{c}\text { Номер } \\
\text { слоя }\end{array}$ & $\begin{array}{c}\text { Оптическая } \\
\text { толщна слоя, } \\
\lambda_{1} / 2 \pi\end{array}$ & $\begin{array}{c}\text { Показатель } \\
\text { преломления }\end{array}$ & $\begin{array}{l}\text { Показатель преломления слоев при ограни- } \\
\text { ченном выборе их значений (4 значения) }\end{array}$ \\
\hline & & 1,74 & 1,61 \\
1 & 1,71 & 2,07 & 2,10 \\
2 & 1,54 & 1,54 & 1,61 \\
3 & 1,33 & 2,45 & 2,45 \\
4 & 1,40 & 1,35 & 1,38 \\
5 & 1,36 & 2,53 & 2,45 \\
6 & 1,30 & 1,49 & 1,38 \\
7 & 1,14 & 2,04 & - \\
8 & 0,97 & 1,84 & 2,10 \\
9 & 1,10 & 2,01 & - \\
10 & 1,26 & 1,60 & 1,61 \\
11 & 1,26 & 2,15 & 2,10 \\
12 & 1,10 & 1,72 & - \\
13 & 0,97 & 1,89 & 1,61 \\
14 & 1,14 & 1,70 & - \\
15 & 1,30 & 2,32 & 2,45 \\
16 & 1,36 & 1,37 & 1,38 \\
17 & 1,40 & 2,59 & 2,45 \\
18 & 1,33 & 1,40 & 1,38 \\
19 & 1,54 & 2,25 & 2,10 \\
20 & 1,71 & 1,68 & 1,61 \\
& & 1,99 & 2,10 \\
& & &
\end{tabular}

ная система, параметры которой приведены в табл. 1. Коэффициент пропускания этой системы показан сплошной линией на рис. 5. Как и ожидалось, мы получили фильтр, сильно отражающий в интервале $\left[0,85 k_{1} ; 1,4 k_{1}\right]$, но имеющий полосы пропускания при волновых числах $0,986 k_{1}$ и $1,171 k_{1}$, причем отношение их равно 1,187 . Относительные ширины $\Delta k / k$ полос пропускания оказываются равными 0,017 и 0,015 . 


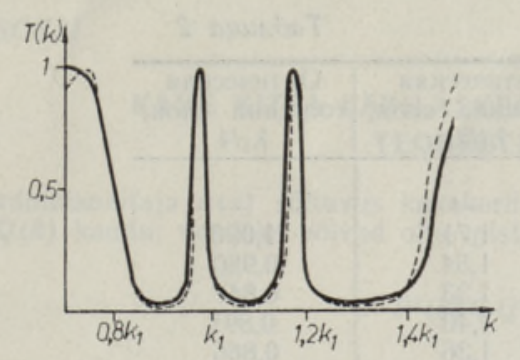

Рис. 5.

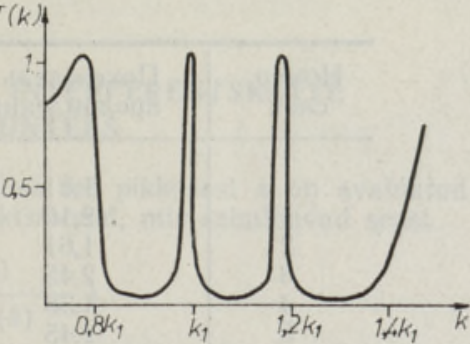

Рис. 6.

В полученном фильтре имеется 22 разлнчных значения показателей преломления. Но так как выбор веществ с различными коэффициентами преломления практически ограничен, то целесообразно осуществлять аппроксимацию с использованием только реальных значений показателей преломления. Выберем четыре значения: $n_{1}=1,38, n_{2}=1,61$, $n_{3}=2,10$ и $n_{4}=2,45$. Этими значениями заменим теперь мало отличающиеся от них другие значения в предыдущей аппроксимации (см. третий столбец в табл. 1). Иными словами, будем проводить аппроксимацию $n(x)$ дискретно-слоистой системой, задав наперед допустимые четыре значения показателей преломления.

Такой подход уменьшает и число слоев, так как уравнивание показателей преломления соседних слоев означает слияние их в один. Фактически в двух местах три слоя объединяются в один. Получается 16-слойная система, коэффициент пропускания которой изображен на рис. 5 прерывистой линией.

Эта характеристика, как оказывается, мало отличается от характеристики предыдущего результата синтеза, где на показатели преломления никаких ограничений не накладывалось. Полосы пропускания находятся теперь около точек $0,9915 k_{1}$ и $1,1800 k_{1}$, причем отношение этих чнсел равно $1,189$.

Рассмотренный пример показывает, что характеристики фильтров данного типа малочувствительны к изменению показателей преломления.

Сделаем еще одно замечание относительно выбора исходной среды. В нашем синтезе ограничивающие среды характеризуются показателями преломления 1,61 и 2,10. Удобнее, однако, иметь в качестве покрытия, наносимого на данную подложку, интерференционную пленку, а в качестве исходной среды - воздух (или вакуум) с показателем преломления 1,00 . Этого можно достичь, превратив одну из ограничивающих сред в дополнительный слой и наложив поверх него просветляющий слой. Сделаем это со стороны среды с показателем преломления 2,10. Получим в результате вместо 16-слойной системы 18-слойную (добавляется слой с показателем преломления 2,10 и просветляющий слой). Спектральная характеристика новой системы выразится формулой

$$
R / T=\frac{R_{1}+R_{2}+2 \sqrt{R_{1} R_{2}} \cos \left[\varphi(k)+2 k n_{17} h\right]}{\sqrt{T_{1} T_{2}}}
$$

(см., напр., $\left.\left[{ }^{3}\right]\right)$, где индекс 1 относится к первоначальной системе, индекс 2 - к просветляющему слою, а $\varphi(k)$ - фазовый множитель, сложным образом зависящий от параметров обеих частей. Так как $R_{2} \approx 0$, то $R / T \approx R_{1} / T_{1}$, т. е. характеристика окончательной просветленной системы мало отличается от характеристики первоначальной. 
Таблица 2

\begin{tabular}{c|c|c|c}
\hline $\begin{array}{c}\text { Номер } \\
\text { слоя }\end{array}$ & $\begin{array}{c}\text { Показатель } \\
\text { преломления }\end{array}$ & $\begin{array}{c}\text { Оптическая } \\
\text { толщина слоя, } \\
\lambda_{1} / 2 \pi\end{array}$ & $\begin{array}{c}\text { Оптическая } \\
\text { толщна слоя, } \\
\lambda_{1} / 4\end{array}$ \\
\hline & 1,61 & - & \\
1 & 2,10 & 1,71 & 1,090 \\
2 & 1,61 & 1,54 & 0,980 \\
3 & 2,45 & 1,33 & 0,847 \\
4 & 1,38 & 1,40 & 0,891 \\
5 & 2,45 & 1,36 & 0,866 \\
6 & 1,38 & 1,30 & 0,828 \\
7 & 2,10 & 3,21 & 2,044 \\
8 & 1,61 & 1,26 & 0,802 \\
9 & 2,10 & 1,26 & 0,802 \\
10 & 1,61 & 3,21 & 2,044 \\
11 & 2,45 & 1,30 & 0,828 \\
12 & 1,38 & 1,36 & 0,866 \\
13 & 2,45 & 1,40 & 0,891 \\
14 & 1,38 & 1,33 & 0,847 \\
15 & 2,10 & 1,54 & 0,980 \\
16 & 1,61 & 1,71 & 1,090 \\
17 & 2,10 & 1,95 & 1,242 \\
18 & 1,38 & 1,44 & 0,917 \\
& 1,00 & - & -
\end{tabular}

Конечно, область максимального просветления необходимо выбрать между полосами пропускания при $k=1,092 k_{1}$. При $n_{17}=2,1$ однослойное просветляющее покрытие должно иметь толщину в четверть длины волны и показатель преломления $\sqrt{2,10}=1,440$. Без значительного ухудшения просветления заменим это значение близким значением $n_{18}=$ $=1,38$. Остается выбрать толщину 17-го слоя. Здесь достаточно использовать метод проб. Как показывают расчеты, искомая толщина оказывает наибольшее влияние на отношение волновых чисел в полосах пропускания. Оно задано нами равным 1,185 .

При толщине просветляющего слоя $1,44 / k_{1}$ наименьшее отклонение отношения частот 1,185 получается при $k_{1} n_{17} h_{17}=1,95$. Полосы пропускания располагаются в этом случае при $0,993 k_{1}$ и $1,180 k_{1}$, а отношение этих чисел равно 1,1878 .

Окончательные результаты даны в табл. 2, где приведены параметры покрытия, и на рис. 6 , где показан коэффициент пропускания $T(k)$. Как видно, покрытие удовлетворяет всем поставленным требованиям. Полосы пропускания имеют относительную ширину 0,013 и 0,012 . Для видимого света ширина полос должна быть порядка 5-10 нм.

\section{Л И ТЕ Р А Т Р А}

1. С ос с и Л., Изв. АН ЭССР, Физ. Матем., 23, № 3, 229-237 (1974).

2. С о с с и Л., Изв. АН ЭССР, Физ. Матем., 26, № 1, 28-36 (1977).

3. К ард П., Анализ и синтез многослойных интерференционных пленок, Таллин, «Валгус», 1971.

Тартуский государственный университет
Поступила в редакцию 24/VI 1977 


\section{KAHE KITSA LABBILASKERIBAGA INTERFERENTSKATTE TEOREETILINE SUNTEES}

Murdumisnäitaja $n(x)$ sõltuvus kahekordsest optilise tee pikkusest $x$ on avaldatud $P(k)$ ja $Q(k)$ kaudu; nendeks vôivad olla mistahes funktsioonid, mis rahuldavad seost

$$
P^{2}(k)+Q^{2}(k)=\frac{1}{T(k)}-1,
$$

kus $T(k)$ kirjeldab läbilaskvuskoefitsiendi sõltuvust lainearvust $k$.

Kitsaste läbilaskeribadega interferentskatete korral on $P(k)$ ja $Q(k)$ kuju analoogiline joonisel 1 esitatuga. Kahe riba korral annavad $P(k)$ ja $Q(k)$ (kirjeldatud valemitega (4), (5) ja joonisel 2) murdumisnäitaja $n(x)(6)$. $n(x)$ on aproksimeeritud diskreetse kõveraga. Saadud 18-kihilise katte parameetrid on toodud tabelis 2.

Katte läbilaskeribad asuvad $k_{1}=0,993 k_{0}$ ja $k_{2}=1,180 k_{0}$ ümbruses, seega $k_{2} / k_{1}=1,1878$ (nõutud oli, et $k_{2} / k_{1}=1,1850$ ). Ribade suhtelised laiused on $\Delta k_{1} / k_{1}=0,013$ ja $\Delta k_{2} / k_{2}=$ $=0,012$.

\section{SOSSI}

\section{THEORETICAL DESIGN OF THE INTERFERENCE COATING WITH TWO NARROW SPECTRAL TRANSMISSION BANDS}

Dependence of the refractive index on the double optical path length $x$ is expressed by $P(k)$ and $Q(k)(1), P(k)$ and $Q(k)$ being any functions which satisfy the equation

$$
P^{2}(k)+Q^{2}(k)=\frac{1}{T(k)}-1,
$$

where $T(k)$ is the transmittance function depending on the wave number $k$. In case of interference coatings with narrow spectral transmission bands, the form of the functions $P(k)$ and $Q(k)$ is similar to that represented in Figure 1. In case of two transmission bands, $P(k)$ and $Q(k)$ being described by the formulas (4), (5) and Figure 2, the refractive index $n(x)$ is given by (6). When $n(x)$ is approximated by a discrete curve, the calculated parameters of an 18-layer coating are shown in Table 2. The coating has transmission bands at $k_{1}=0.993 k_{0}$ and $k_{2}=1.180 k_{0}$, therefore $k_{2} / k_{1}=1.1878$ (the required quotient was $\left.k_{2} / k_{1}=1.1850\right)$. Transmission intervals are $\Delta k_{1} / k_{1}=0.013$ and $\Delta k_{2} / k_{2}=0.012$. 